\title{
TENSOR PRODUCT OF DUALIZING COMPLEXES OVER A FIELD
}

\author{
LIRAN SHAUL
}

\begin{abstract}
Let $\mathbb{k}$ be a field, and let $X, Y$ be two locally noetherian $\mathbb{k}$-schemes (respectively $\mathbb{k}$-formal schemes) with dualizing complexes $R_{X}$ and $R_{Y}$ respectively. We show that $R_{X} \bigotimes_{\mathbb{k}} R_{Y}$ (respectively its derived completion) is a dualizing complex over $X \times_{\mathbb{K}} Y$ if and only if $X \times_{\mathbb{k}} Y$ is locally noetherian of finite Krull dimension.
\end{abstract}

\section{CONTENTS}

0. Introduction

1. Tensor product of dualizing complexes over ordinary schemes

2. Tensor product of dualizing complexes over formal schemes

References

\section{INTRODUCTION}

Throughout this note, rings are assumed to be commutative and unital. Given a ring $A$, we denote by $\mathrm{D}(\operatorname{Mod} A)$ the derived category of $A$-modules, and by $\mathrm{D}^{\mathrm{b}}(\operatorname{Mod} A)$ and $\mathrm{D}_{\mathrm{f}}^{\mathrm{b}}(\operatorname{Mod} A)$ its triangulated subcategories made of bounded complexes, and bounded complexes with coherent cohomology respectively. We will also use commutative DG-algebras. Given such a DG-algebra $A$, we will denote the category of differential graded $A$-modules by $\mathrm{DGMod} A$, and its derived category by $\mathrm{D}(\operatorname{DGMod} A)$.

Dualizing complexes, first introduced in [RD] half a century ago, are now a ubiquitous tool in commutative algebra and algebraic geometry. In this note we are concerned with dualizing complexes over a fibre product of schemes or formal schemes over a field.

In the first section we work with ordinary schemes. Our main result in Section 1 shows that if two locally noetherian schemes $X, Y$, over a field $\mathbb{k}$, have dualizing complexes $R_{X}, R_{Y}$, then the only obstruction for $X \times_{\mathbb{k}} Y$ to possess a dualizing complex is the trivial one, namely, $X \times_{\mathbb{k}} Y$ must be locally noetherian, and of finite Krull dimension. In that case we show that the box tensor product $R_{X} \bigotimes_{\mathbb{k}} R_{Y}$ is a dualizing complex over $X \times_{\mathbb{k}} Y$. This is proven in Corollary 1.7 below. If the schemes involved are of finite type over $\mathbb{k}$, then this is not new, and could be easily deduced from the results of [RD]. In fact, in that case one can even replace $\mathbb{k}$ by a Gorenstein ring, assume one of $X, Y$ is flat over it, and replace tensor product with derived tensor product. We however make no finiteness assumption on either of the maps $X \rightarrow \mathbb{k}, Y \rightarrow \mathbb{k}$.

One interesting consequence of this result in the affine case, given in Corollary 1.8 below, is the fact that for such noetherian rings, the tensor product functor $-\otimes_{\mathbb{k}}-$ : $\mathrm{D}_{\mathrm{f}}^{\mathrm{b}}(\operatorname{Mod} A) \times \mathrm{D}_{\mathrm{f}}^{\mathrm{b}}(\operatorname{Mod} B) \rightarrow \mathrm{D}_{\mathrm{f}}^{\mathrm{b}}\left(\operatorname{Mod} A \otimes_{\mathbb{k}} B\right)$ preserves finite injective dimension.

The author acknowledges the support of the European Union for the ERC grant No 257004-HHNcdMir. 
In section 2 we switch to the more difficult case of formal schemes. We are able to reproduce the above result in the formal case, and prove that if $\mathfrak{X}$ and $\mathfrak{Y}$ are two locally noetherian formal schemes over a field $\mathbb{k}$, with dualizing complex $R_{\mathfrak{X}}$ and $R_{\mathfrak{Y}}$ respectively, and if $\mathfrak{X} \times \mathbb{R} \mathfrak{Y}$ is locally noetherian and of finite Krull dimension, then the derived completion and derived torsion of $R_{\mathscr{X}} \bigotimes_{\mathbb{K}} R_{\mathscr{Y}}$ are c-dualizing and t-dualizing complexes (notions that are recalled in Section 2 below) over $\mathfrak{X} \times_{\mathbb{k}} \mathfrak{Y}$. This is given in Theorem 2.9 below.

To understand why the formal case is much more involved, consider the simplest corresponding affine situation, where $\mathbb{k}$ is a field, and $A$ and $B$ are two noetherian Gorenstein $\mathbb{k}$-algebras of finite Krull dimension, which are adically complete with respect to some ideals $\mathfrak{a} \subseteq A$ and $\mathfrak{b} \subseteq B$. The Gorenstein hypothesis implies that $A$ and $B$ are dualizing complexes over themselves, so what we need to prove is that in this situation, the completed tensor product $A \widehat{\otimes_{\mathbb{k}}} B$ is also a Gorenstein ring, whenever it is noetherian of finite Krull dimension. However, the ring $A \otimes_{\mathbb{k}} B$ is usually non-noetherian, so we do not know if the completion map $A \otimes_{\mathbb{k}} B \rightarrow A \widehat{\otimes_{\mathbb{k}}} B$ is flat, and so we do not know if in general the maps $A \rightarrow A \widehat{\otimes}_{\mathbb{k}} B$ and $B \rightarrow A \widehat{\otimes}_{\mathbb{k}} B$ are flat. This rules out attempts to prove such a result using the methods used in the corresponding discrete case (i.e, when $\mathfrak{a}=0$ and $\mathfrak{b}=0$, so that $A \otimes_{\mathbb{k}} B$ is noetherian) given in [TY] and other similar papers. As a replacement for flatness, we rely heavily on the theory of weakly proregular ideals of [AJL1, Sc, PSY1]. Using it and some other homological and homotopical tools, we are able to prove the above mentioned result about dualizing complexes over fiber product of formal schemes.

Acknowledgments. The author would like to thank Amnon Yekutieli for some useful suggestions.

\section{TENSOR PRODUCT OF DUALIZING COMPLEXES OVER ORDINARY SCHEMES}

We shall need the following result, which is contained in the proof of [ $\mathrm{Ka}$, Corollary $1.4]$.

Proposition 1.1. Let $A$ be a commutative noetherian ring. Assume that $A$ has a dualizing complex. Then there is a finite type A-algebra $A^{\prime}$ which is Gorenstein of finite Krull dimension, and such that there is a surjection $A^{\prime} \rightarrow A$.

Proof. By [ $\mathrm{Ka}$, Corollary 1.4], there is a Gorenstein ring $A^{\prime}$ of finite Krull dimension, and a surjection $A^{\prime} \rightarrow A$, so it is enough to verify that this ring is a finitely generated $A$ algebra. The first step in the proof of [ Ka Corollary 1.4] reduces to the case where the codimension function of $A$ is constant on its associated primes. One way to do this is using [ $\mathrm{Ka}$, Lemma 5.5], which says that if a $\operatorname{ring} A$ is noetherian, universally catenary, and has a codimension function (all these properties are satisfied by a ring possessing a dualizing complex), then there exist a finite type $A$-algebra $B$ whose codimension function is constant on its associated primes, and such that there is a surjection $B \rightarrow A$. Hence, we may assume without loss of generality that the codimension function of our $A$ is constant on its associated primes. Next, for such an $A$, it is shown in [Ka], that there some ideal $I \subseteq A$ of positive height, such that the Rees algebra $A(I)=\oplus_{n \geq 0}(I x)^{n} \subseteq A[x]$ is Cohen-Macaulay. Note that if $I=\left(f_{1}, \ldots, f_{m}\right)$, then the map $A\left[x_{1}, \ldots, x_{m}\right] \rightarrow A(I)$ given by $x_{i} \mapsto f_{i} \cdot x$ is surjective, so that $A(I)$ is of finite type over $A$. As $A$ is a quotient of $A(I)$, we reduce to the case where $A$ is Cohen-Macaulay. Then, in the final step of [ $\mathrm{Ka}$, Corollary 1.4], it is observed that by [Sh, Theorem 4.3], there is some finitely generated $A$-module $M$, such that the trivial extension ring $A^{\prime}:=A \times M$ (in the sense of [Ma Chapter 25, page 191]) is a finite dimensional Gorenstein ring. As there is a surjection $A^{\prime} \rightarrow A$, and as $A^{\prime}$ is clearly a finite type $A$-algebra, we are done. 
Lemma 1.2. Let $\mathbb{k}$ be a field, and let $A$ and $B$ be two noetherian $\mathbb{k}$-algebras with dualizing complexes $R_{A}$ and $R_{B}$ respectively, such that $A \otimes_{\mathbb{k}} B$ is a noetherian ring. Then the canonical map

$$
A \otimes_{\mathbb{k}} B \rightarrow \mathrm{RHom}_{A \otimes_{\mathbb{k}} B}\left(R_{A} \otimes_{\mathbb{k}} R_{B}, R_{A} \otimes_{\mathbb{k}} R_{B}\right)
$$

is an isomorphism in $\mathrm{D}\left(\operatorname{Mod} A \otimes_{\mathbb{k}} B\right)$.

Proof. Since $R_{A}$ and $R_{B}$ are dualizing complexes, they have finitely generated bounded cohomologies, and in particular we may assume that they are bounded. Hence, by [YZ, Lemma 8.4], there is an isomorphism

$$
\mathrm{RHom}_{A}\left(R_{A}, R_{A}\right) \otimes_{\mathbb{k}} \operatorname{RHom}_{B}\left(R_{B}, R_{B}\right) \cong \mathrm{RHom}_{A \otimes_{\mathbb{k}} B}\left(R_{A} \otimes_{\mathbb{k}} R_{B}, R_{A} \otimes_{\mathbb{k}} R_{B}\right)
$$

in $\mathrm{D}\left(\operatorname{Mod} A \otimes_{\mathbb{k}} B\right)$. Again, the fact that $R_{A}$ and $R_{B}$ are dualizing complexes implies that

$$
\mathrm{RHom}_{A}\left(R_{A}, R_{A}\right) \otimes_{\mathbb{k}} \operatorname{RHom}_{B}\left(R_{B}, R_{B}\right) \cong A \otimes_{\mathbb{R}} B .
$$

Composing these two isomorphisms, we deduce that there is some isomorphism

$$
\mathrm{RHom}_{A \otimes_{\mathbb{k}} B}\left(R_{A} \otimes_{\mathbb{k}} R_{B}, R_{A} \otimes_{\mathbb{k}} R_{B}\right) \cong A \otimes_{\mathbb{k}} B
$$

in $\mathrm{D}\left(\operatorname{Mod} A \otimes_{\mathbb{k}} B\right)$. Hence, by an unpublished result of Foxby, given in AIL, Proposition 2.3], the canonical map

$$
A \otimes_{\mathbb{k}} B \rightarrow \mathrm{RHom}_{A \otimes_{\mathbb{k}} B}\left(R_{A} \otimes_{\mathbb{k}} R_{B}, R_{A} \otimes_{\mathbb{k}} R_{B}\right)
$$

is also an isomorphism.

The following lemma is probably well known. We reproduce its easy proof for the convenience of the reader:

Lemma 1.3. Let $A$ be a noetherian ring, and let $R$ be a dualizing complex over $A$. A complex $M \in \mathrm{D}_{\mathrm{f}}^{\mathrm{b}}(\operatorname{Mod} A)$ has finite injective dimension over $A$ if and only if the complex $\mathrm{RHom}_{A}(M, R)$ is perfect.

Proof. Since $\mathrm{RHom}_{A}(M, R)$ has finitely generated cohomologies, by [AF, Corollary 2.10.F], it is perfect if and only if it has finite flat dimension, and by [RD, Proposition V.2.6], this happens if and only if

$$
\mathrm{RHom}_{A}\left(\mathrm{RHom}_{A}(M, R), R\right) \cong M
$$

has finite injective dimension over $A$.

Lemma 1.4. Let $\mathbb{k}$ be a field, and let $A$ and $B$ be two noetherian $\mathbb{k}$-algebras, such that $A \otimes_{\mathbb{k}}$ $B$ is also noetherian. Assume that there are dualizing complexes $R_{A}$ over $A$ and $R_{B}$ over $B$, such that $R_{A} \otimes_{\mathbb{k}} R_{B}$ is a dualizing complex over $A \otimes_{\mathbb{k}} B$. Then for any dualizing complex $S_{A}$ over $A$, and any dualizing complex $S_{B}$ over $B$, the complex $S_{A} \otimes_{\mathbb{k}} S_{B}$ is a dualizing complex over $A \otimes_{\mathbb{k}} B$.

Proof. By Lemma1.2, the canonical map

$$
A \otimes_{\mathbb{k}} B \rightarrow \operatorname{RHom}_{A \otimes_{\mathbb{k}} B}\left(S_{A} \otimes_{\mathbb{k}} S_{B}, S_{A} \otimes_{\mathbb{k}} S_{B}\right)
$$

is an isomorphism, so it is enough to show that $S_{A} \otimes_{\mathbb{k}} S_{B}$ has finite injective dimension over $A \otimes_{\mathbb{k}} B$. Since by assumption $R_{A} \otimes_{\mathbb{k}} R_{B}$ is a dualizing complex, by Lemma 1.3 it is enough to show that the complex

$$
\mathrm{R} \operatorname{Hom}_{A \otimes_{\mathbb{k}} B}\left(S_{A}, \otimes_{\mathbb{k}} S_{B}, R_{A} \otimes_{\mathbb{k}} R_{B}\right)
$$

is perfect, but this is clear, since by [YZ, Lemma 8.4], it is isomorphic to

$$
\mathrm{RHom}_{A}\left(S_{A}, R_{A}\right) \otimes_{\mathbb{k}} \mathrm{RHom}_{B}\left(S_{B}, R_{B}\right),
$$


and because the (box) tensor product of two finitely generated projectives is a finitely generated projective.

In the next lemma, we will have to use differential graded algebras, and dualizing DG-modules over them. We refer the reader to [YYe2] for the terminology regarding DGalgebras used in this lemma. The lemma essentially says that if for a pair of $\mathbb{k}$-algebras $A^{\prime}, B^{\prime}$, our main theorem about tensor product of dualizing complexes holds, then it also holds for any pair of quotients $A^{\prime} \rightarrow A, B^{\prime} \rightarrow B$.

Lemma 1.5. Let $\mathbb{k}$ be a field, and let $A^{\prime}, B^{\prime}$ be two noetherian $\mathbb{k}$-algebras with dualizing complexes $R_{A}$ and $R_{B}$ respectively. Assume that $R_{A} \otimes_{\mathbb{R}} R_{B}$ is a dualizing complex over the noetherian ring $A^{\prime} \otimes_{\mathbb{k}} B^{\prime}$. Let $A$ be an $A^{\prime}$-algebra, and let $B$ be a $B^{\prime}$-algebra such that the structure maps $A^{\prime} \rightarrow A$ and $B^{\prime} \rightarrow B$ are surjective. Then

$$
\mathrm{RHom}_{A^{\prime}}\left(A, R_{A}\right) \otimes_{\mathbb{R}} \mathrm{RHom}_{B^{\prime}}\left(B, R_{B}\right)
$$

is a dualizing complex over $A \otimes_{\mathbb{k}} B$.

Proof. Since the map $A^{\prime} \otimes_{\mathbb{R}} B^{\prime} \rightarrow A \otimes_{\mathbb{k}} B$ is finite, it follows from [RD, Proposition V.2.4] that

$$
\mathrm{RHom}_{A^{\prime} \otimes_{\mathbb{k}} B^{\prime}}\left(A \otimes_{\mathbb{k}} B, R_{A} \otimes_{\mathbb{k}} R_{B}\right)
$$

is a dualizing complex over $A \otimes_{\mathbb{k}} B$. By [YZ, Lemma 8.4], there is an isomorphism

$$
\mathrm{RHom}_{A^{\prime}}\left(A, R_{A}\right) \otimes_{\mathbb{k}} \mathrm{RHom}_{B^{\prime}}\left(B, R_{B}\right) \cong \operatorname{RHom}_{A^{\prime} \otimes_{\mathbb{k}} B^{\prime}}\left(A \otimes_{\mathbb{k}} B, R_{A} \otimes_{\mathbb{k}} R_{B}\right)
$$

obtained by replacing $A$ and $B$ by projective resolutions over $A^{\prime}$ and $B^{\prime}$ respectively. However, this isomorphism is only $A^{\prime} \otimes_{\mathbb{k}} B^{\prime}$-linear, and the author does not know how to show that there is such an $A \otimes_{\mathbb{k}} B$-linear isomorphism. Instead, using [Av, Proposition 2.2.8] let $A^{\prime} \rightarrow \widetilde{A} \cong A$ and $B^{\prime} \rightarrow \widetilde{B} \cong B$ be DG-algebra resolutions of $A^{\prime} \rightarrow A$ and $B^{\prime} \rightarrow B$ respectively, such that $\widetilde{A}^{0}=A^{\prime}$ and $\widetilde{B}^{0}=B^{\prime}$, for each $i<0, \widetilde{A}^{i}$ is a finitely generated projective $\widetilde{A}^{0}$-module, $\widetilde{B}^{i}$ is a finitely generated projective $\widetilde{B}^{0}$-module, and for each $i>0, \widetilde{A}^{i}=\widetilde{B}^{i}=0$. Then, as shown in the proof of [YZ, Lemma 8.4], the natural map

$$
\operatorname{Hom}_{A^{\prime}}\left(\widetilde{A}, R_{A}\right) \otimes_{\mathbb{k}} \operatorname{Hom}_{B^{\prime}}\left(\widetilde{B}, R_{B}\right) \rightarrow \operatorname{Hom}_{A^{\prime} \otimes_{\mathbb{k}} B^{\prime}}\left(\widetilde{A} \otimes_{\mathbb{k}} \widetilde{B}, R_{A} \otimes_{\mathbb{k}} R_{B}\right)
$$

which is clearly $\widetilde{A} \otimes_{\mathbb{R}} \widetilde{B}$-linear, is an isomorphism. Since $\widetilde{A}$ is K-projective over $A^{\prime}, \widetilde{B}$ is K-projective over $B^{\prime}$, and $\widetilde{A} \otimes_{\mathbb{k}} \widetilde{B}$ is K-projective over $A^{\prime} \otimes_{\mathbb{k}} B^{\prime}$, we deduce that there is an isomorphism

$$
\mathrm{RHom}_{A^{\prime}}\left(\widetilde{A}, R_{A}\right) \otimes_{\mathbb{k}} \operatorname{RHom}_{B^{\prime}}\left(\widetilde{B}, R_{B}\right) \cong \operatorname{RHom}_{A^{\prime} \otimes_{\mathbb{k}} B^{\prime}}\left(\widetilde{A} \otimes_{\mathbb{k}} \widetilde{B}, R_{A} \otimes_{\mathbb{k}} R_{B}\right)
$$

in $\mathrm{D}\left(\operatorname{DGMod} \widetilde{A} \otimes_{\mathbb{k}} \widetilde{B}\right)$. By [Ye2, Proposition 7.5(1)], the right hand side is a dualizing DGmodule over $\widetilde{A} \otimes_{\mathbb{k}} \widetilde{B}$, so that, the left hand side is also a dualizing DG-module. As there are isomorphisms

$$
\mathrm{RHom}_{A^{\prime}}\left(\widetilde{A}, R_{A}\right) \cong \operatorname{RHom}_{A^{\prime}}\left(A, R_{A}\right)
$$

and

$$
\mathrm{RHom}_{B^{\prime}}\left(\widetilde{B}, R_{B}\right) \cong \mathrm{RHom}_{B^{\prime}}\left(B, R_{B}\right)
$$

over $\widetilde{A}$ and $\widetilde{B}$ respectively, it follows that the DG-module

$$
\mathrm{RHom}_{A^{\prime}}\left(A, R_{A}\right) \otimes_{\mathbb{R}} \mathrm{RHom}_{B^{\prime}}\left(B, R_{B}\right)
$$

is a dualizing DG-module over $\widetilde{A} \otimes_{\mathbb{k}} \widetilde{B}$. Set $R=\mathrm{RHom}_{A^{\prime}}\left(A, R_{A}\right) \otimes_{\mathbb{k}} \operatorname{RHom}_{B^{\prime}}\left(B, R_{B}\right) \in$ $\mathrm{D}\left(\operatorname{Mod} A \otimes_{\mathbb{k}} B\right)$. Because $\widetilde{A} \otimes_{\mathbb{k}} \widetilde{B} \rightarrow A \otimes_{\mathbb{k}} B$ is a quasi-isomorphism, the fact that the image of $R$ in the derived category over $\widetilde{A} \otimes_{\mathbb{k}} \widetilde{B}$ has a finite injective dimension implies that $R$ has finite injective dimension over $A \otimes_{\mathbb{k}} B$. By Lemma 1.2 , the canonical map $A \otimes_{\mathbb{k}} B \rightarrow$ 
$\mathrm{RHom}_{A \otimes_{\mathbb{k}} B}(R, R)$ is an isomorphism. Hence, $R=\mathrm{R}_{\operatorname{Hom}_{A^{\prime}}}\left(A, R_{A}\right) \otimes_{\mathbb{k}} \mathrm{RHom}_{B^{\prime}}\left(B, R_{B}\right)$ is a dualizing complex over $A \otimes_{\mathbb{k}} B$.

We now give the main result of this section.

Theorem 1.6. Let $\mathbb{k}$ be a field, and let $A, B$ be commutative noetherian $\mathbb{k}$-algebras. Assume that $A$ and $B$ have dualizing complexes. Then the ring $A \otimes_{\mathbb{k}} B$ has a dualizing complex if and only if $A \otimes_{\mathbb{R}} B$ is noetherian of finite Krull dimension. In that case, for every dualizing complexes $R_{A}$ over $A$ and $R_{B}$ over $B$, the complex $R_{A} \otimes_{\mathbb{k}} R_{B}$ is a dualizing complex over $A \otimes_{\mathbb{k}} B$.

Proof. The only if part is well known. Assume that $A \otimes_{\mathbb{k}} B$ is noetherian of finite Krull dimension. Let $A^{\prime} \rightarrow A$ and $B^{\prime} \rightarrow B$ be the Gorenstein rings guaranteed to exist from Proposition 1.1 Since $A \otimes_{\mathbb{k}} B$ is noetherian of finite Krull dimension, and since $A^{\prime}$ (respectively $B^{\prime}$ ) is a finite type $A$ (resp. $B$ )-algebra, it follows that $A^{\prime} \otimes_{\mathbb{k}} B^{\prime}$ is also noetherian of finite Krull dimension. Hence, by [TY, Theorem 6(a)], the ring $A^{\prime} \otimes_{\mathbb{k}} B^{\prime}$ is also Gorenstein. Let $R:=\mathrm{RHom}_{A^{\prime}}\left(A, A^{\prime}\right)$, and let $S:=\mathrm{RHom}_{B^{\prime}}\left(B, B^{\prime}\right)$. As $A^{\prime}$ is Gorenstein of finite Krull dimension, $A^{\prime}$ is a dualizing complex over $A^{\prime}$, so that by [RD, Proposition V.2.4] $R$ is a dualizing complex over $A$, and in the same manner, $S$ is a dualizing complex over $B$. Similarly, since $A^{\prime} \otimes_{\mathbb{R}} B^{\prime}$ is Gorenstein of finite Krull dimension, $A^{\prime} \otimes_{\mathbb{R}} B^{\prime}$ is a dualizing complex over $A^{\prime} \otimes_{\mathbb{k}} B^{\prime}$. Thus, the conditions of Lemma 1.5 are satisfied for $A^{\prime} \rightarrow A$ and $B^{\prime} \rightarrow B$, so that

$$
\mathrm{RHom}_{A^{\prime}}\left(A, A^{\prime}\right) \otimes_{\mathbb{k}} \mathrm{R} \operatorname{Hom}_{B^{\prime}}\left(B, B^{\prime}\right)=R \otimes_{\mathbb{k}} S
$$

is a dualizing complex over $A \otimes_{\mathbb{k}} B$. Hence, by Lemma 1.4 , the same is true for the complex $R_{A} \otimes_{\mathbb{k}} R_{B}$.

Since one can check the property of being a dualizing complex on an affine open cover (because of [RD Lemma II.7.16]), we obtain:

Corollary 1.7. Let $\mathbb{k}$ be a field, and let $X, Y$ be two locally noetherian $\mathbb{k}$-schemes with dualizing complexes $R_{X}$ and $R_{Y}$ respectively. If $X \times_{\mathbb{k}} Y$ is locally noetherian of finite Krull dimension, then $R_{X} \bigotimes_{\mathbb{R}} R_{Y}$ is a dualizing complex over $X \times_{\mathbb{k}} Y$.

Corollary 1.8. Let $\mathbb{k}$ be a field, and let $A, B$ be two $\mathbb{k}$-algebras. Assume that $A$ and $B$ have dualizing complexes $R_{A}$ and $R_{B}$ respectively, and that $A \otimes_{\mathbb{R}} B$ is noetherian of finite Krull dimension. Given a complex $M \in \mathrm{D}_{\mathrm{f}}^{\mathrm{b}}(\operatorname{Mod} A)$ and a complex $N \in \mathrm{D}_{\mathrm{f}}^{\mathrm{b}}(\operatorname{Mod} B)$, if $M$ has finite injective dimension over $A$, and $N$ has finite injective dimension over $B$, then $M \otimes_{\mathbb{k}} N$ has finite injective dimension over $A \otimes_{\mathbb{R}} B$.

Proof. By Lemma 1.3, the complexes $\mathrm{RHom} A\left(M, R_{A}\right)$ and $\mathrm{R} \operatorname{Hom}_{B}\left(N, R_{B}\right)$ are perfect over $A$ and $B$ respectively. Hence, the complex $\operatorname{RHom}_{A}\left(M, R_{A}\right) \otimes_{\mathbb{k}} \mathrm{RHom}_{B}\left(N, R_{B}\right)$ is perfect over $A \otimes_{\mathbb{k}} B$. By [YZ, Lemma 8.4], there is an isomorphism

$$
\mathrm{R}_{\operatorname{Hom}}\left(M, R_{A}\right) \otimes_{\mathbb{k}} \operatorname{RHom}_{B}\left(N, R_{B}\right) \cong \operatorname{RHom}_{A \otimes_{\mathbb{k}} B}\left(M \otimes_{\mathbb{k}} N, R_{A} \otimes_{\mathbb{k}} R_{B}\right),
$$

and since by Theorem $1.6, R_{A} \otimes_{\mathbb{k}} R_{B}$ is a dualizing complex over $A \otimes_{\mathbb{k}} B$, we have that

$$
M \otimes_{\mathbb{k}} N \cong \mathrm{RHom}_{A \otimes_{\mathbb{k}} B}\left(\mathrm{RHom}_{A \otimes_{\mathbb{k}} B}\left(M \otimes_{\mathbb{k}} N, R_{A} \otimes_{\mathbb{k}} R_{B}\right), R_{A} \otimes_{\mathbb{k}} R_{B}\right)
$$

so the result follows from applying Lemma 1.3 again.

Remark 1.9. The fact that Corollary 1.8 follows from the theorem about tensor product of dualizing complexes was already observed in [YZ, Corollary 8.6], in a noncommutative situation. The result given there, in the commutative setting, makes the assumption that both $A$ and $B$ are finitely generated $\mathbb{k}$-algebras. 


\section{TENSOR PRODUCT OF DUALIZING COMPLEXES OVER FORMAL SCHEMES}

We now turn to generalize Theorem 1.6 to formal schemes. To do that, we first recall some adic homological algebra. We refer the reader to [AJL1, AJL2, PSY1, PSY2, Sc] for a detailed treatment of the material below. By a preadic ring $(A, \mathfrak{a})$, we shall mean a commutative ring $A$ equipped with an adic topology generated by some finitely generated ideal $\mathfrak{a} \subseteq A$ (It is important to note that we do not assume that $A$ is noetherian). Given a preadic $\operatorname{ring}(A, \mathfrak{a})$, there are functors $\Gamma_{\mathfrak{a}}(-):=\lim \operatorname{Hom}_{A}\left(A / \mathfrak{a}^{n},-\right)$ and $\Lambda_{\mathfrak{a}}(-):=\lim A / \mathfrak{a}^{n} \otimes_{A}-$ called the $\mathfrak{a}$-torsion and $\mathfrak{a}$-completion functors. These are both additive functors $\operatorname{Mod} A \rightarrow$ $\operatorname{Mod} A$. The $A$-module $\widehat{A}:=\Lambda_{\mathfrak{a}}(A)$ has a structure of a commutative ring, and there is a natural map $A \rightarrow \widehat{A}$. If this map is bijective then we will call $(A, \mathfrak{a})$ an adic ring, and say that $A$ is a-adically complete. For any $M \in \operatorname{Mod} A$, the $A$-modules $\Gamma_{\mathfrak{a}}(M)$ and $\Lambda_{\mathfrak{a}}(M)$ naturally carry a $\widehat{A}$-module structure, so that we obtain functors $\widehat{\Gamma}_{\mathfrak{a}}, \widehat{\Lambda}_{\mathfrak{a}}: \operatorname{Mod} A \rightarrow \operatorname{Mod} \widehat{A} \operatorname{defined}$ by exactly the same formulas as $\Gamma_{\mathfrak{a}}$ and $\Lambda_{\mathfrak{a}}$. The derived functors

$$
\mathrm{R} \Gamma_{\mathfrak{a}}, \mathrm{L} \Lambda_{\mathfrak{a}}: \mathrm{D}(\operatorname{Mod} A) \rightarrow \mathrm{D}(\operatorname{Mod} A)
$$

and

$$
\mathrm{R} \widehat{\Gamma}_{\mathfrak{a}}, \mathrm{L} \widehat{\Lambda}_{\mathfrak{a}}: \mathrm{D}(\operatorname{Mod} A) \rightarrow \mathrm{D}(\operatorname{Mod} \widehat{A})
$$

exist. $R \Gamma_{\mathfrak{a}}$ and $\mathrm{R} \widehat{\Gamma}_{\mathfrak{a}}$ are calculated using K-injective resolutions, while $L \Lambda_{\mathfrak{a}}$ and $\mathrm{L} \widehat{\Lambda}_{\mathfrak{a}}$ are calculated using K-flat resolutions. See [AJL1, Section 1] for a proof.

For any $M \in \mathrm{D}(\operatorname{Mod} A)$, there are canonical morphisms $\mathrm{R} \Gamma_{\mathfrak{a}}(M) \rightarrow M$ and $M \rightarrow \mathrm{L} \Lambda_{\mathfrak{a}}(M)$ in $\mathrm{D}(\operatorname{Mod} A)$. If these maps are isomorphisms we say that $M$ is cohomologically a-torsion and cohomologically $\mathfrak{a}$-adically complete respectively. The collections of all cohomologically $\mathfrak{a}$-torsion and cohomologically $\mathfrak{a}$-adically complete complexes form two full triangulated subcategories of $\mathrm{D}(\operatorname{Mod} A)$. These are denoted by $\mathrm{D}(\operatorname{Mod} A)_{\mathfrak{a}-\operatorname{tor}}$ and $\mathrm{D}(\operatorname{Mod} A)_{\mathfrak{a}-\operatorname{com}}$ respectively.

Given a ring $A$ and a finite sequence of elements $\mathbf{a}$, there is a bounded complex of free $A$-modules, $\operatorname{Tel}(A ; \mathbf{a})$ called the telescope complex associated to a. See [PSY1, Section 5] for its definition. If $A \rightarrow B$ is a ring homomorphism, and if $\mathbf{b}$ is the image of $\mathbf{a}$ under this map, then there is an isomorphism of complexes $\operatorname{Tel}(A ; \mathbf{a}) \otimes_{A} B \rightarrow \operatorname{Tel}(B ; \mathbf{b})$. Given an ideal $\mathfrak{a} \subseteq A$, and a finite sequence of elements $\mathbf{a} \subseteq A$ that generates $\mathfrak{a}$, there is a morphism of functors $\mathrm{R} \Gamma_{\mathfrak{a}}(-) \rightarrow \operatorname{Tel}(A ; \mathbf{a}) \otimes_{A}-$. If this morphism is a quasi-isomorphism then a and $\mathfrak{a}$ are said to be weakly proregular. See [Sc, Section 2], and in particular [Sc, Definition 2.3]. In a noetherian ring, every ideal and every finite sequence are weakly proregular. If the ideal $\mathfrak{a}$ is weakly proregular, then the functors

$$
\begin{aligned}
\mathrm{R} \Gamma_{\mathfrak{a}}: \mathrm{D}(\operatorname{Mod} A)_{\mathfrak{a} \text {-com }} & \rightarrow \mathrm{D}(\operatorname{Mod} A)_{\mathfrak{a} \text {-tor }}, \\
\mathrm{L} \Lambda_{\mathfrak{a}}: \mathrm{D}(\operatorname{Mod} A)_{\mathfrak{a} \text {-tor }} & \rightarrow \mathrm{D}(\operatorname{Mod} A)_{\mathfrak{a} \text {-com }}
\end{aligned}
$$

are quasi-inverse to each other, and induce an equivalence between these two triangulated categories, called the Matlis-Greenlees-May equivalence. If $A$ is noetherian and a-adically complete, then

$$
\mathrm{D}_{\mathrm{f}}^{\mathrm{b}}(\operatorname{Mod} A) \subseteq \mathrm{D}(\operatorname{Mod} A)_{\mathfrak{a}-\operatorname{com}} .
$$

In that case, the essential image of $\mathrm{D}_{\mathrm{f}}^{\mathrm{b}}(\operatorname{Mod} A)$ under the functor $\mathrm{R} \Gamma_{\mathfrak{a}}$ is denoted by $\mathrm{D}^{\mathrm{b}}(\operatorname{Mod} A)_{\mathfrak{a}-\text { cof }}$. This is a triangulated category, called the category of cohomologically $\mathfrak{a}$-adically cofinite complexes, and is equivalent to the category $\mathrm{D}_{\mathrm{f}}^{\mathrm{b}}(\operatorname{Mod} A)$. See [PSY2] for a study of this category.

The following proposition, whose proof is immediate from the definitions will be useful in the sequel. 
Proposition 2.1. Let $A$ be a commutative ring, let $\mathfrak{a} \subseteq A$ be an ideal, and let $\widehat{A}:=\Lambda_{\mathfrak{a}}(A)$. Let $Q: \mathrm{D}(\operatorname{Mod} \widehat{A}) \rightarrow \mathrm{D}(\operatorname{Mod} A)$ be the forgetful functor. Then there are isomorphisms

$$
Q \circ \mathrm{L} \widehat{\Lambda}_{\mathfrak{a}}(-) \cong \mathrm{L} \Lambda_{\mathfrak{a}}(-)
$$

and

$$
Q \circ \mathrm{R} \widehat{\Gamma}_{\mathfrak{a}}(-) \cong \mathrm{R} \Gamma_{\mathfrak{a}}(-)
$$

of functors $\mathrm{D}(\operatorname{Mod} A) \rightarrow \mathrm{D}(\operatorname{Mod} A)$.

2.1. A reduction lemma. The aim of this subsection is to prove Lemma 2.5, which allows us to reduce certain questions over $\widehat{A}$ to questions over $A$.

The next lemma was inspired by a result of Yekutieli (private communication).

Lemma 2.2. Let $A \rightarrow B$ be a quasi-isomorphism of commutative $D G$-algebras. Let $C$ be a $B$-algebra, and let $Q: \mathrm{D}(\operatorname{DGMod} B) \rightarrow \mathrm{D}(\operatorname{DGMod} A)$ be the forgetful functor. Then there is an isomorphism

$$
\mathrm{RHom}_{B}(C,-) \cong \mathrm{RHom}_{A}(C, Q(-))
$$

of functors $\mathrm{D}(\mathrm{DGMod} B) \rightarrow \mathrm{D}(\operatorname{Mod} C)$.

Proof. Let $M \in \mathrm{D}(\mathrm{DGMod} B)$. Let $M \rightarrow I_{B}$ be a K-injective resolution of $M$ over $B$, and let $Q(M) \rightarrow I_{A}$ be a K-injective resolution of $M$ over $A$. The functor $Q$ induces an isomorphism $I_{B} \cong I_{A}$ in $\mathrm{D}(\operatorname{DGMod} A)$. Since $I_{A}$ is $\mathrm{K}$-injective, there is some $A$-linear quasi-isomorphism $\alpha: I_{B} \rightarrow I_{A}$. Composition with $\alpha$ induces a map $\alpha^{\prime}: \operatorname{Hom}_{B}\left(C, I_{B}\right) \rightarrow \operatorname{Hom}_{A}\left(C, I_{A}\right)$, and this map is clearly $C$-linear. It is enough to show that $\alpha^{\prime}$ is a quasi-isomorphism. To see this, consider the map $\phi: I_{B} \rightarrow \operatorname{Hom}_{A}\left(B, I_{A}\right)$ given by $\phi(x)(b)=\alpha(b \cdot x)$, for $b \in B$ and $x \in I_{B}$. This map fits into a commutative diagram

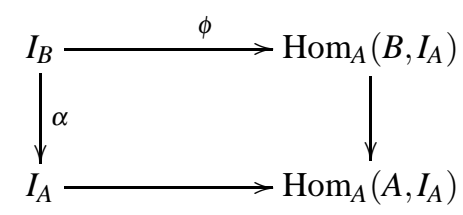

Because $A \rightarrow B$ is a quasi-isomorphism, and $I_{A}$ is K-injective, the right vertical map is a quasi-isomorphism. Hence, $\phi$ is also a quasi-isomorphism between two K-injective DG $B$-modules, so it is a homotopy equivalence. Hence, in the commutative diagram

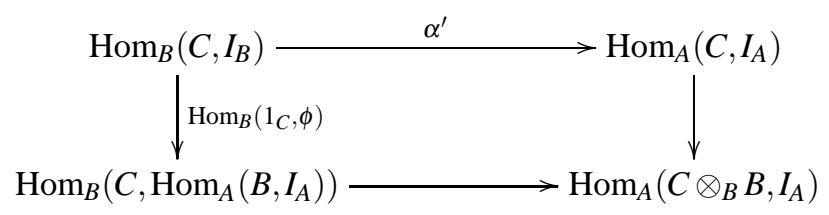

the left vertical arrow induced by this homotopy equivalence is a quasi-isomorphism, while the right vertical arrow and the bottom horizontal arrow are obviously isomorphisms. Hence, $\alpha^{\prime}$ is a quasi-isomorphism, as claimed.

Lemma 2.3. Let $A$ be a commutative ring, let $\mathfrak{a} \subseteq A$ be a finitely generated weakly proregular ideal, and set $\widehat{A}:=\Lambda_{\mathfrak{a}}(A)$. Let $J \subseteq A$ be an ideal, and assume that there are integers $m, n$, such that $\mathfrak{a}^{m} \subseteq J \subseteq \mathfrak{a}^{n}$. Then for all $i \neq 0$, we have that

$$
\operatorname{Tor}_{i}^{A}(A / J, \widehat{A})=0 \text {. }
$$


Proof. Let $B=A / J$. Let $\mathbf{a}$ be a finite sequence of elements that generates $\mathfrak{a}$, and let $\mathbf{b}$ be its image in $B$. Note that by assumption, each element of $\mathbf{b}$ is nilpotent. Hence, by [PSY1, Lemma 7.4], there is a $B$-linear homotopy equivalence $\operatorname{Tel}(B ; \mathbf{b}) \rightarrow B$. By the base change property of the telescope complex, we deduce that there is an $A$-linear homotopy equivalence $\operatorname{Tel}(A ; \mathbf{a}) \otimes_{A} B \rightarrow B$.

Since $\mathfrak{a}$ is weakly proregular, by the Greenlees-May duality (specifically, by [AJL1, Item (iv) of Corollary after Theorem (0.3)*], or by [PSY1, Lemma 7.6]), there is an isomorphism $\widehat{A} \otimes_{A} \operatorname{Tel}(A ; \mathbf{a}) \cong \operatorname{Tel}(A ; \mathbf{a})$ in $\mathrm{D}(\operatorname{Mod} A)$.

Combining these two isomorphisms, and the fact that $\operatorname{Tel}(A ; \mathbf{a})$ is a $\mathrm{K}$-flat complex, we obtain the following sequence of isomorphisms in $\mathrm{D}(\operatorname{Mod} A)$ :

$$
B \cong \operatorname{Tel}(A ; \mathbf{a}) \otimes_{A}^{\mathrm{L}} B \cong\left(\widehat{A} \otimes_{A}^{\mathrm{L}} \operatorname{Tel}(A ; \mathbf{a})\right) \otimes_{A}^{\mathrm{L}} B \cong \widehat{A} \otimes_{A}^{\mathrm{L}}\left(\operatorname{Tel}(A ; \mathbf{a}) \otimes_{A}^{\mathrm{L}} B\right) \cong \widehat{A} \otimes_{A}^{\mathrm{L}} B .
$$

As $B$ is a complex concentrated in degree 0 , the result follows.

Lemma 2.4. Let $A$ be a commutative ring, let $\mathfrak{a} \subseteq A$ be a finitely generated weakly proregular ideal, and set $\widehat{A}:=\Lambda_{\mathfrak{a}}(A)$. Let $J \subseteq A$ be an ideal, and assume that there are integers $m, n$, such that $\mathfrak{a}^{m} \subseteq J \subseteq \mathfrak{a}^{n}$. Set $B=A / J$, and let $Q_{A}: \mathrm{D}(\operatorname{Mod} \widehat{A}) \rightarrow \mathrm{D}(\operatorname{Mod} A)$ be the forgetful functor. Then there is an isomorphism

$$
\mathrm{RHom}_{\widehat{A}}(B,-) \cong \mathrm{RHom}_{A}\left(B, Q_{A}(-)\right)
$$

of functors $\mathrm{D}(\operatorname{Mod} \widehat{A}) \rightarrow \mathrm{D}(\operatorname{Mod} B)$.

Proof. Let $A \rightarrow \widetilde{A} \cong \widehat{A}$ be a commutative semi-free DG-algebra resolution of $A \rightarrow \widehat{A}$, and let $Q_{\widetilde{A}}: \mathrm{D}(\operatorname{Mod} \widehat{A}) \rightarrow \mathrm{D}(\operatorname{DGMod} \widetilde{A})$ be the corresponding forgetful functor. Given $M \in$ $\mathrm{D}(\operatorname{Mod} \widehat{A})$, according to Lemma2.2 there is an isomorphism of functors

$$
\mathrm{RHom}_{\widehat{A}}(B, M) \cong \operatorname{RHom}_{\tilde{A}}\left(B, Q_{\widetilde{A}}(M)\right) .
$$

Let $Q_{\widetilde{A}}(M) \rightarrow I$ be a K-injective resolution of $Q_{\widetilde{A}}(M)$ over $\widetilde{A}$. Then there is an obvious $B$-linear isomorphism

$$
\operatorname{RHom}_{\widetilde{A}}\left(B, Q_{\widetilde{A}}(M)\right) \cong \operatorname{Hom}_{\widetilde{A}}(B, I) .
$$

According to lemma 2.3, we have that $\operatorname{Tor}_{i}^{A}(B, \widehat{A})=0$ for all $i \neq 0$. Hence, the map $B \otimes_{A}$ $\widetilde{A} \rightarrow B \otimes_{A} \widehat{A}$ induced by the map $\widetilde{A} \rightarrow \widehat{A}$ is a quasi-isomorphism.

Since $\mathfrak{a}^{m} \subseteq J$, we have that $B \otimes_{A} A / \mathfrak{a}^{m} \cong B$. On the other hand, since $\mathfrak{a}$ is finitely generated, we have that $A / \mathfrak{a}^{m} \otimes_{A} \widehat{A} \cong A / \mathfrak{a}^{m}$. Combining these two facts, we deduce that $B \otimes_{A} \widehat{A} \cong B$. It follows that there is a quasi-isomorphism $B \otimes_{A} \widetilde{A} \rightarrow B$, which is $B$-linear on the left, and $\widetilde{A}$-linear on the right 1

This in turn induces a quasi-isomorphism

$$
\operatorname{Hom}_{\widetilde{A}}(B, I) \rightarrow \operatorname{Hom}_{\widetilde{A}}\left(B \otimes_{A} \widetilde{A}, I\right),
$$

which by the hom-tensor adjunction is naturally isomorphic to

$$
\operatorname{Hom}_{A}(B, I) \text {. }
$$

Since $A \rightarrow \widetilde{A}$ is flat, we deduce that $I$ is $\mathrm{K}$-injective over $A$, so that $\operatorname{Hom}_{A}(B, I) \cong$ $\mathrm{RHom}_{A}\left(B, Q_{A}(M)\right)$, which proves the claim.

\footnotetext{
${ }^{1}$ The main reason we needed the to take the DG-algebra resolution $A \rightarrow \widetilde{A} \cong \widehat{A}$ was in order to get these linearity conditions on this quasi-isomorphism. These allow us now to use the hom-tensor adjunction. The fact that there is such an $A$-linear isomorphism is already proved in Lemma2.3 but this fact is not enough to use adjunction in the next step of the proof.
} 
Lemma 2.5. Let $A$ be a commutative ring, let $\mathfrak{a} \subseteq A$ be a finitely generated weakly proregular ideal, and set $\widehat{A}:=\Lambda_{\mathfrak{a}}(A)$. Let $J \subseteq A$ be an ideal, and assume that there are integers $m, n$, such that $\mathfrak{a}^{m} \subseteq J \subseteq \mathfrak{a}^{n}$. Set $B=A / J$. Then there are isomorphisms

$$
\mathrm{RHom}_{\widehat{A}}\left(B, \mathrm{R}_{\mathfrak{a}}(-)\right) \cong \operatorname{RHom}_{\widehat{A}}\left(B, \operatorname{L}_{\mathfrak{a}}(-)\right) \cong \operatorname{RHom}_{A}(B,-)
$$

of functors $\mathrm{D}(\operatorname{Mod} A) \rightarrow \mathrm{D}(\operatorname{Mod}(B)$.

Proof. Let $Q: \mathrm{D}(\operatorname{Mod} \widehat{A}) \rightarrow \mathrm{D}(\operatorname{Mod} A)$ be the forgetful functor. According to Lemma2.4 there are $B$-linear isomorphisms of functors

$$
\operatorname{RHom}_{\widehat{A}}\left(B, \mathrm{R} \widehat{\Gamma}_{\mathfrak{a}}(-)\right) \cong \operatorname{RHom}_{A}\left(B, Q\left(\mathrm{R} \widehat{\Gamma}_{\mathfrak{a}}(-)\right)\right)
$$

and

$$
\operatorname{RHom}_{\widehat{A}}\left(B, \mathrm{~L} \widehat{\Lambda}_{\mathfrak{a}}(-)\right) \cong \operatorname{RHom}_{A}\left(B, Q\left(\mathrm{~L} \widehat{\Lambda}_{\mathfrak{a}}(-)\right)\right)
$$

By Proposition 2.1, these are isomorphic in $\mathrm{D}(\operatorname{Mod} B)$ to

$$
\mathrm{RHom}_{A}\left(B, \mathrm{R} \Gamma_{\mathfrak{a}}(-)\right)
$$

and

$$
\mathrm{RHom}_{A}\left(B, \mathrm{~L} \Lambda_{\mathfrak{a}}(-)\right)
$$

respectively. In the proof of Lemma 2.3 we have seen that $B \cong \operatorname{Tel}(A ; \mathbf{a}) \otimes_{A} B$, which implies that $B$ is cohomologically $\mathfrak{a}$-torsion. Hence, by the Greenlees-May duality (AJL1, Theorem 0.3], or [PSY1, Theorem 7.12], there are natural isomorphisms

$$
\mathrm{RHom}_{A}\left(B, \mathrm{R} \Gamma_{\mathfrak{a}}(-)\right) \cong \mathrm{R} \operatorname{Hom}_{A}(B,-) \cong \mathrm{RHom}_{A}\left(B, \mathrm{~L} \Lambda_{\mathfrak{a}}(-)\right) .
$$

The isomorphisms constructed in [AJL1, PSY1] are $A$-linear, but it is easy to verify that in our situation they are actually $B$-linear. This proves the claim.

2.2. The box tensor products over affine formal schemes. Next, we obtain some general finiteness results about the adic box tensor products. For a moment, we drop the assumption that $\mathbb{k}$ is a field, as it does not produce additional difficulties, and it seems that this result might be of independent interest in this greater generality.

Proposition 2.6. Let $\mathbb{k}$ be a commutative ring, and let $(A, \mathfrak{a})$ and $(B, \mathfrak{b})$ be two noetherian adic rings which are flat $\mathbb{k}$-algebras. Let $I=\mathfrak{a} \otimes_{\mathbb{k}} B+A \otimes_{\mathbb{k}} \mathfrak{b}$ be the ideal of definition of the adic topology on $A \otimes_{\mathbb{k}} B$, let $\widehat{I}$ be the ideal generated by its image in $\widehat{A \otimes_{\mathbb{R}} B}$, and assume that I is weakly proregular (if $\mathbb{k}$ is a field this always holds), and that $\widehat{A \otimes_{\mathbb{k}} B}$ is noetherian. Given $M \in \mathrm{D}_{\mathrm{f}}^{\mathrm{b}}(\operatorname{Mod} A)$ and $N \in \mathrm{D}_{\mathrm{f}}^{\mathrm{b}}(\operatorname{Mod} B)$ with $M$ having finite flat dimension over $\mathbb{k}$, we have that

and

$$
\mathrm{L} \widehat{\Lambda}_{I}\left(M \otimes_{\mathbb{k}}^{\mathrm{L}} N\right) \in \mathrm{D}_{\mathrm{f}}^{\mathrm{b}}\left(\operatorname{Mod} \widehat{A \otimes_{\mathbb{k}} B}\right),
$$

$$
\mathrm{R} \widehat{\Gamma}_{I}\left(M \otimes_{\mathbb{k}}^{\mathrm{L}} N\right) \in \mathrm{D}^{\mathrm{b}}\left(\operatorname{Mod} \widehat{A \otimes_{\mathbb{k}} B}\right)_{\widehat{I}-\operatorname{cof}} .
$$

Proof. We first show that both of these complexes have bounded cohomology. Let $Q$ : $\mathrm{D}\left(\operatorname{Mod} \widehat{A \otimes_{\mathbb{k}} B}\right) \rightarrow \mathrm{D}\left(\operatorname{Mod} A \otimes_{\mathbb{k}} B\right)$ be the forgetful functor. Clearly, a complex $X$ has bounded cohomology if and only if the complex $Q(X)$ has bounded cohomology. In view of Proposition 2.1, it is enough to show that the complexes

$$
\mathrm{L} \Lambda_{I}\left(M \otimes_{\mathbb{k}}^{\mathrm{L}} N\right)
$$

and

$$
\mathrm{R}_{I}\left(M \otimes_{\mathbb{k}}^{\mathrm{L}} N\right)
$$

have bounded cohomology, but this follows immediately from the flat dimension assumption on $M$, combined with the fact that when $I$ is weakly proregular, the functors $\mathrm{L} \Lambda_{I}$ and 
$\mathrm{R} \Gamma_{I}$ have finite cohomological dimension (for example, by [PSY1, Corollary 4.28] and [PSY1, Corollary 5.27]).

Next, we show the claims about finiteness of the cohomologies. Let $P \rightarrow M$ and $Q \rightarrow N$ be bounded above resolutions made of finitely generated free modules. As $A$ is flat over $\mathbb{k}, P$ is also flat over $\mathbb{k}$, so that $M \otimes_{\mathbb{k}}^{\mathrm{L}} N \cong P \otimes_{\mathbb{k}} Q$, and the latter is also a bounded above complex made of finitely generated free modules, so that

$$
\mathrm{L} \widehat{\Lambda}_{I}\left(M \otimes_{\mathbb{k}}^{\mathrm{L}} N\right) \cong \Lambda_{I}\left(P \otimes_{\mathbb{k}} Q\right) .
$$

Since the completion functor commutes with finite direct sums, it follows that $\Lambda_{I}\left(P \otimes_{\mathbb{k}} Q\right)$ is also a bounded above complex made of finitely generated free modules, which shows that the cohomologies of this complex are finitely generated over $\widehat{A \otimes_{\mathbb{k}} B}$. It remains to show that

$$
\mathrm{R} \widehat{\Gamma}_{I}\left(M \otimes_{\mathbb{k}}^{\mathrm{L}} N\right) \in \mathrm{D}^{\mathrm{b}}\left(\operatorname{Mod} \widehat{A \otimes_{\mathbb{k}} B}\right)_{\widehat{I}-\operatorname{cof}} .
$$

As we already established that this complex is bounded, and as it is clearly cohomologically $\widehat{I}$-torsion, by [PSY2, Theorem 3.10], it is enough to show that the complex

$$
\mathrm{RHom}_{\widehat{A \otimes_{\mathbb{k}} B}}\left(\widehat{A \otimes_{\mathbb{k}} B} / \widehat{I}, \widehat{R}_{I}\left(M \otimes_{\mathbb{k}}^{\mathrm{L}} N\right)\right)
$$

has finitely generated cohomologies. By Lemma 2.5, there is an isomorphism

$$
\mathrm{RHom}_{\widehat{A \otimes_{\mathbb{k}} B}}\left(\widehat{A \otimes_{\mathbb{k}} B} / \widehat{I}, \widehat{R}_{\Gamma_{I}}\left(M \otimes_{\mathbb{k}}^{\mathrm{L}} N\right)\right) \cong \operatorname{RHom}_{\widehat{A \otimes_{\mathbb{k}} B}}\left(\widehat{A \otimes_{\mathbb{k}} B} / \widehat{I}, \mathrm{~L} \widehat{\Lambda}_{I}\left(M \otimes_{\mathbb{k}}^{\mathrm{L}} N\right)\right),
$$

so the result follows from the first claim in this proposition.

Remark 2.7. One might wonder why in the above proof we had to invoke the rather difficult theorem of [PSY2], instead of deducing the finiteness condition in the torsion case directly from the identity $\mathrm{R} \widehat{\Gamma}_{I}(-) \cong \mathrm{R} \Gamma_{\widehat{I}^{\circ}} \mathrm{L} \widehat{\Lambda}_{I}(-)$. The reason for that is that we do not know if this identity holds when $A \otimes_{\mathbb{k}} B \rightarrow \widehat{A \otimes_{\mathbb{k}} B}$ is not flat.

2.3. Tensor product of dualizing complexes over formal schemes. In this subsection we will prove Theorem 2.9, the main result of this section. First, we recall the definitions of dualizing complexes over affine formal schemes. See [AJL2, Section 2.5] and [Ye1, Section 5] for details (keeping in mind [PSY2, Theorem 3.10]). Let $(A, \mathfrak{a})$ be an adic noetherian ring. A complex $R \in \mathrm{D}(\operatorname{Mod} A)$ which has finite injective dimension over $A$, and such that the canonical map $A \rightarrow \mathrm{RHom}_{A}(R, R)$ is an isomorphism is called a c-dualizing complex if $R \in \mathrm{D}_{\mathrm{f}}^{\mathrm{b}}(\operatorname{Mod} A)$, and is called a t-dualizing complex if $R \in \mathrm{D}^{\mathrm{b}}(\operatorname{Mod} A)_{\mathfrak{a}-\operatorname{cof}}$.

The next lemma allows us to reduce the problem of determining if a complex over the completed tensor product is dualizing to a problem over discrete rings. We will then use Theorem 1.6 to obtain the required result.

Lemma 2.8. Let $\mathbb{k}$ be a field, and let $(A, \mathfrak{a})$ and $(B, \mathfrak{b})$ be two noetherian adic rings which are $\mathbb{k}$-algebras, such that the completed tensor product $\widehat{A \otimes_{\mathbb{k}} B}$ is noetherian of finite Krull dimension. Let I be the ideal of definition of the adic topology on $\widehat{A \otimes_{\mathbb{R}} B}$, and let $M \in$ $\mathrm{D}_{\mathrm{f}}^{\mathrm{b}}\left(\operatorname{Mod} \widehat{A \otimes_{\mathbb{k}} B}\right)$ (respectively $\left.M \in \mathrm{D}\left(\operatorname{Mod} \widehat{A \otimes_{\mathbb{k}} B}\right)_{\mathrm{I}-\mathrm{cof}}\right)$. Then $M$ is a c-dualizing (resp. $t$-dualizing) complex over $\widehat{A \otimes_{\mathbb{R}} B}$ if and only if for each $n>0$ the complex

$$
\mathrm{R} \operatorname{Hom}_{\widehat{A \otimes_{\mathbb{k}} B}}\left(A / \mathfrak{a}^{n} \otimes_{\mathbb{k}} B / \mathfrak{b}^{n}, M\right) \in \mathrm{D}\left(\operatorname{Mod} A / \mathfrak{a}^{n} \otimes_{\mathbb{k}} B / \mathfrak{b}^{n}\right)
$$

is a dualizing complex over $A / \mathfrak{a}^{n} \otimes_{\mathbb{R}} B / \mathfrak{b}^{n}$. 
Proof. Consider the sequence of ideals $J_{n}=\operatorname{ker}\left(\widehat{A \otimes_{\mathbb{k}} B} \rightarrow A / \mathfrak{a}^{n} \otimes_{\mathbb{k}} B / \mathfrak{b}^{n}\right)$. For every $n \in$ $\mathbb{N}$, there is some $m \in \mathbb{N}$, such that $J_{n} \subseteq I^{m}$, and likewise, for every $n \in \mathbb{N}$, there is some $m \in \mathbb{N}$, such that $I^{n} \subseteq J_{m}$. Hence,

$$
\lim _{\longleftarrow}\left(\widehat{A \otimes_{\mathbb{k}} B} / J_{n}\right) \cong \widehat{A \otimes_{\mathbb{k}} B}
$$

and moreover, the two functors $\Gamma_{I}(-)$ and $\lim _{\longrightarrow} \operatorname{Hom}_{\widehat{A \otimes_{\mathrm{k}} B}}\left(\widehat{A \otimes_{\mathbb{k}} B} / J_{n},-\right)$ are canonically isomorphic. With these observations, the result now follows from the proof of [AJL2, Lemma 2.5.10] (see also [Fa, Satz 2]).

We now arrive to the main result of this section, an adic generalization of Theorem 1.6

Theorem 2.9. Let $\mathbb{k}$ be a field, and let $(A, \mathfrak{a})$ and $(B, \mathfrak{b})$ be two noetherian adic rings which are $\mathbb{k}$-algebras. Let $I$ be the ideal of definition of the adic topology on $A \otimes_{\mathbb{k}} B$. Let $R_{A}$ be a $c$-dualizing complex over $(A, \mathfrak{a})$, and let $R_{B}$ be a c-dualizing complex over $(B, \mathfrak{b})$. Then the ring $\widehat{A \otimes_{\mathbb{k}} B}$ has dualizing complexes if and only if it is noetherian of finite Krull dimension. In that case, $\mathrm{L} \widehat{\Lambda}_{I}\left(R_{A} \otimes_{\mathbb{R}} R_{B}\right)$ is a c-dualizing complex over $\widehat{A \otimes_{\mathbb{R}} B}$, and $\mathrm{R} \widehat{\Gamma}_{I}\left(R_{A} \otimes_{\mathbb{R}} R_{B}\right)$ is at-dualizing complex over $\widehat{A \otimes_{\mathbb{k}} B}$.

Proof. Let $\widehat{I}$ be the ideal generated by the image of $I$ in $\widehat{A \otimes_{\mathbb{k}} B}$. According to [PSY1, Example 4.35], the ideal $I$ is weakly proregular. Hence, by Proposition 2.6, we have that

$$
\mathrm{L} \widehat{\Lambda}_{I}\left(R_{A} \otimes_{\mathbb{k}} R_{B}\right) \in \mathrm{D}_{\mathrm{f}}^{\mathrm{b}}\left(\operatorname{Mod} \widehat{A \otimes_{\mathbb{k}} B}\right),
$$

and

$$
\mathrm{R} \widehat{\Gamma}_{I}\left(R_{A} \otimes_{\mathbb{k}} R_{B}\right) \in \mathrm{D}^{\mathrm{b}}\left(\operatorname{Mod} \widehat{A \otimes_{\mathbb{k}} B}\right)_{\widehat{I}-\text { cof }}
$$

By Lemma 2.8, it is enough to show that for all $n$, the complexes

$$
\mathrm{RHom}_{\widehat{A \otimes_{\mathbb{k}} B}}\left(A / \mathfrak{a}^{n} \otimes_{\mathbb{k}} B / \mathfrak{b}^{n}, \mathrm{~L} \widehat{\Lambda}_{I}\left(R_{A} \otimes_{\mathbb{k}} R_{B}\right)\right)
$$

and

$$
\mathrm{R} \operatorname{Hom}_{\widehat{A \otimes_{\mathbb{k}} B}}\left(A / \mathfrak{a}^{n} \otimes_{\mathbb{k}} B / \mathfrak{b}^{n}, \mathrm{R} \widehat{\Gamma}_{I}\left(R_{A} \otimes_{\mathbb{k}} R_{B}\right)\right)
$$

are dualizing complexes over $A / \mathfrak{a}^{n} \otimes_{\mathbb{k}} B / \mathfrak{b}^{n}$. By Lemma 2.5, both of these complexes are isomorphic as objects in $\mathrm{D}\left(\operatorname{Mod} A / \mathfrak{a}^{n} \otimes_{\mathbb{k}} B / \mathfrak{b}^{n}\right)$, and moreover, both of them are isomorphic to the complex

$$
\mathrm{R} \operatorname{Hom}_{A \otimes_{\mathbb{k}} B}\left(A / \mathfrak{a}^{n} \otimes_{\mathbb{k}} B / \mathfrak{b}^{n}, R_{A} \otimes_{\mathbb{k}} R_{B}\right)
$$

Note that as the maps $A \rightarrow A / \mathfrak{a}^{n}$ and $B \rightarrow B / \mathfrak{b}^{n}$ are finite, the complexes

$$
\mathrm{RHom}_{A}\left(A / \mathfrak{a}^{n}, R_{A}\right)
$$

and

$$
\mathrm{RHom}_{B}\left(B / \mathfrak{b}^{n}, R_{B}\right)
$$

are dualizing complexes over $A / \mathfrak{a}^{n}$ and $B / \mathfrak{b}^{n}$ respectively. Since the $\operatorname{ring} A / \mathfrak{a}^{n} \otimes_{\mathbb{k}} B / \mathfrak{b}^{n}$ is noetherian of finite Krull dimension (being a quotient of the noetherian ring of finite Krull dimension $\left.\widehat{A \otimes_{\mathbb{k}} B}\right)$, it follows from Theorem 1.6 that

$$
\operatorname{RHom}_{A}\left(A / \mathfrak{a}^{n}, R_{A}\right) \otimes_{\mathbb{k}} \operatorname{RHom}_{B}\left(B / \mathfrak{b}^{n}, R_{B}\right)
$$

is a dualizing complex over $A / \mathfrak{a}^{n} \otimes_{\mathbb{k}} B / \mathfrak{b}^{n}$. We now use the same trick as in the proof of Lemma 1.5. Thus, let $A \rightarrow \widetilde{A} \cong A / \mathfrak{a}^{n}$ and $B \rightarrow \widetilde{B} \cong B / \mathfrak{b}^{n}$ be DG-algebra resolutions 
of $A \rightarrow A / \mathfrak{a}^{n}$ and $B \rightarrow B / \mathfrak{b}^{n}$ respectively as in Lemma 1.5 . Then there is a $\widetilde{A} \otimes_{\mathbb{R}} \widetilde{B}$-linear isomorphism

$$
\begin{array}{r}
\operatorname{RHom}_{A \otimes_{\mathbb{k}} B}\left(A / \mathfrak{a}^{n} \otimes_{\mathbb{k}} B / \mathfrak{b}^{n}, R_{A} \otimes_{\mathbb{k}} R_{B}\right) \cong \\
\operatorname{RHom}_{A}\left(A / \mathfrak{a}^{n}, R_{A}\right) \otimes_{\mathbb{k}} \operatorname{RHom}_{B}\left(B / \mathfrak{b}^{n}, R_{B}\right) .
\end{array}
$$

The right hand side is a dualizing complex over $A / \mathfrak{a}^{n} \otimes_{\mathbb{k}} B / \mathfrak{b}^{n}$, and hence, also a dualizing DG-module over $\widetilde{A} \otimes_{\mathbb{k}} \widetilde{B}$. Hence, the left hand side, which is a priori a complex over $A / \mathfrak{a}^{n} \otimes_{\mathbb{k}} B / \mathfrak{b}^{n}$ is also a dualizing DG-module over $\widetilde{A} \otimes_{\mathbb{k}} \widetilde{B}$. Hence, by the argument used in the proof of Lemma 1.5, we deduce that

$$
\mathrm{R} \operatorname{Hom}_{A \otimes_{\mathbb{k}} B}\left(A / \mathfrak{a}^{n} \otimes_{\mathbb{R}} B / \mathfrak{b}^{n}, R_{A} \otimes_{\mathbb{R}} R_{B}\right)
$$

is a dualizing complex over $A / \mathfrak{a}^{n} \otimes_{\mathbb{R}} B / \mathfrak{b}^{n}$, which establishes the theorem.

Again, as in Corollary 1.7, this generalizes immediately to formal schemes. As an immediate corollary, we obtain an adic generalization of [TY, Theorem 6(a)].

Corollary 2.10. Let $\mathbb{k}$ be a field, and let $(A, \mathfrak{a})$ and $(B, \mathfrak{b})$ be two adic noetherian Gorenstein $\mathbb{k}$-algebras of finite Krull dimension, such that $\widehat{A \otimes_{\mathbb{k}} B}$ is also noetherian of finite Krull dimension. Then $\widehat{A \otimes_{\mathbb{k}} B}$ is also a Gorenstein ring.

Remark 2.11. As far as we know, all similar results in the literature concerning the conservation of homological properties of commutative noetherian rings under the tensor product operation involves a flatness assumption. In that sense, the above Corollary is different, because, to our knowledge, it is not known if in the above situation the maps $A \rightarrow \widehat{A \otimes_{\mathbb{k}} B}$ and $B \rightarrow \widehat{A \otimes_{\mathbb{k}} B}$ are flat (because it is not known if the completion map $A \otimes_{\mathbb{k}} B \rightarrow \widehat{A \otimes_{\mathbb{k}} B}$ is flat when $A \otimes_{\mathbb{k}} B$ is non-noetherian), although, flatness is known to hold if $A / \mathfrak{a}$ is essentially of finite type over $\mathbb{k}$ (See [AJL2, Proposition 7.1(b)]). We thus view this result as another example of the fact that weak proregularity of the ideal of definition of the adic topology can serve as a replacement for flatness of the completion map in many interesting situations.

Cohen structure theorem may be stated as follows: given a noetherian local ring $(A, \mathfrak{m})$, its completion $\Lambda_{\mathfrak{m}}(A)$ is a quotient of a regular local ring. Our final corollary is a weak variation of it for tensor product of local rings. It says that the completion of a tensor product of local rings is a quotient of a Gorenstein ring.

Corollary 2.12. Let $\mathbb{k}$ be a field, and let $(A, \mathfrak{m})$ and $(B, \mathfrak{n})$ be two noetherian local $\mathbb{k}-$ algebras. Let $I=\mathfrak{m} \otimes_{\mathbb{k}} B+A \otimes_{\mathbb{k}} \mathfrak{n}$, and assume that

$$
\widehat{A \otimes_{\mathbb{k}} B}:=\Lambda_{I}\left(A \otimes_{\mathbb{k}} B\right)
$$

is noetherian of finite Krull dimension. Then $\widehat{A \otimes_{\mathbb{R}} B}$ has dualizing complexes, so it is a quotient of a Gorenstein ring of finite Krull dimension.

Proof. By Cohen structure theorem, the rings $\widehat{A}$ and $\widehat{B}$ have dualizing complexes. Since there are isomorphisms $A / \mathfrak{m}^{n} \cong \widehat{A} /(\mathfrak{m} \cdot \widehat{A})^{n}$ and $B / \mathfrak{n}^{n} \cong \widehat{B} /(\mathfrak{n} \cdot \widehat{B})^{n}$, we see as in the proof of Lemma 2.8 that

$$
\widehat{A \otimes_{\mathbb{k}} B} \cong \lim _{\longleftarrow}\left(A / \mathfrak{m}^{n} \otimes_{\mathbb{k}} B / \mathfrak{n}^{n}\right) \cong \lim _{\longleftarrow}\left(\widehat{A} /(\mathfrak{m} \cdot \widehat{A})^{n} \otimes_{\mathbb{k}} \widehat{B} /(\mathfrak{n} \cdot \widehat{B})^{n}\right) \cong \widehat{\widehat{A} \otimes_{\mathbb{k}} \widehat{B}}
$$


where $\widehat{\widehat{A} \otimes_{\mathbb{k}} \widehat{B}}:=\Lambda_{J}\left(\widehat{A} \otimes_{\mathbb{k}} \widehat{B}\right), J:=(\mathfrak{m} \cdot \widehat{A}) \otimes_{\mathbb{k}} \widehat{B}+\widehat{A} \otimes_{\mathbb{k}}(\mathfrak{n} \cdot \widehat{B})$. By Theorem 2.9, the ring $\widehat{\widehat{A} \otimes_{\mathbb{k}} \widehat{B}}$ has dualizing complexes, so the isomorphic ring $\widehat{A \otimes_{\mathbb{k}} B}$ also has dualizing complexes. Hence, by Kawasaki's theorem, it is a quotient of a Gorenstein ring of finite Krull dimension.

\section{REFERENCES}

[Av] Avramov, L. L. (1998). Infinite free resolutions. In Six lectures on commutative algebra (pp. 1-118). Birkhauser Basel.

[AF] Avramov, L. L., and Foxby, H. B. (1991). Homological dimensions of unbounded complexes. Journal of Pure and Applied Algebra, 71(2), 129-155.

[AIL] Avramov, L. L., Iyengar, S., and Lipman, J. (2010). Reflexivity and rigidity for complexes, I: Commutative rings. Algebra and Number Theory, 4(1), 47-86.

[AJL1] Alonso Tarrio, L., Jeremias Lopez, A., and Lipman, J. (1997). Local homology and cohomology on schemes. In Annales scientifiques de l'Ecole normale superieure (Vol. 30, No. 1, pp. 1-39).

[AJL2] Alonso Tarrio, L., Jeremias Lopez, A., and Lipman, J. (1999). Duality and flat base change on formal schemes. Studies in Duality on Noetherian formal schemes and non-Noetherian ordinary schemes, Contemporary Mathematics, 244, 1-87.

[Fa] Faltings, G. (1978). Zur Existenz dualisierender Komplexe. Mathematische Zeitschrift, 162(1), 7586.

[Ka] Kawasaki, T. (2002). On arithmetic Macaulayfication of Noetherian rings. Transactions of the American Mathematical Society, 354(1), 123-149.

[Ma] Matsumura, H. (1989). Commutative ring theory (Vol. 8). Cambridge university press.

[PSY1] Porta, M., Shaul, L., and Yekutieli, A. (2014). On the homology of completion and torsion. Algebras and Representation Theory, 17(1), 31-67.

[PSY2] Porta, M., Shaul, L., and Yekuiteli A. (2015), Cohomologically cofinite complexes, Comm. Algebra, 43:2, 597-615.

[RD] Hartshorne, R. "Residues and Duality," Lecture Notes in Math. 20, Springer-Verlag, Berlin, 1966.

[Sc] Schenzel, P. Proregular sequences, Local Cohomology, and Completion, Math. Scand. 92 (2003), 161- 180.

[Sh] Sharp, R. Y. (1979). Necessary conditions for the existence of dualizing complexes in commutative algebra. Séminaire d'Algèbre Paul Dubreil, Proceedings, Paris 1977-78, Lecture Notes in Mathematics, vol. 740, Springer, Berlin, 1979, pp. 213-229.

[TY] Tousi, M., and Yassemi, S. (2003). Tensor products of some special rings. Journal of Algebra, 268(2), 672-676.

[Ye1] Yekutieli, A. (1998). Smooth formal embeddings and the residue complex. Canadian Journal of Mathematics, 50(4), 863.

[Ye2] Yekutieli, A. (2013). Duality and Tilting for Commutative DG Rings. arXiv preprint arXiv:1312.6411 2 .

[YZ] Yekutieli, A., and Zhang, J. J. (2005). Dualizing complexes and perverse modules over differential algebras. Compositio Mathematica, 141(03), 620-654.

Universiteit Antwerpen, Departement Wiskunde-Informatica, Middelheim Campus, MidDELHEIMLAAN 1, 2020 ANTWERP, BELGIUM

E-mail address: Liran. Shaul@uantwerpen.be 